\title{
UTERINE RUPTURE DURING PREGNANCY AND DELIVERY AMONG WOMEN ATTENDING THE AL-TTHAWRA HOSPITAL IN SANA'A CITY YEMEN REPUBLIC
}

\author{
Ishraq Dhaifalah, $^{\mathrm{a}, \mathrm{b}}$, Jiri Santavy ${ }^{\mathrm{b}}$, Helena Fingerova ${ }^{\mathrm{c}}$
}

\author{
${ }^{a}$ Department of Human Genetic and Fetal Medicine, Faculty of Medicine and Dentistry, Palacky University and University \\ Hospital, Olomouc, Czech Republic \\ ${ }^{b}$ Department of Obstetric and Gynecology, Al-thawra Hospital, Sana'a University, Sana'a, Yemen Republic \\ Department of Obstetric and Gynecology, University Hospital, Olomouc \\ e-mail: ishraq_dhaifalah@yahoo.com
}

Received: April 28, 2006; Accepted: June 6, 2006

Key words: Rupture uterus/Incidence/Causes/Maternal mortality and morbidity/Prenatal mortality and morbidity/ Yemen

Background: About 20 percent of the population in developing countries is composed of women of reproductive age. These women face one of the catastrophic risks of pregnancy "uterine rupture”. Studies conducted in the developing world give strong evidence that uterine rupture is a major health problem in these countries with the rate being high in rural areas.

Aim: The purpose of the study was to estimate the incidence and determine the risk factors and outcome of uterine rupture among women using the referral hospital Al-thawra in Sana'a City, Yemen republic and to extrapolate the data to the whole of Yemen.

Methods: The data was collected retrospectively; by interviewing, examining and following up all the cases of uterine rupture coming to the hospital during a period of 9 months between September 1996 and May 1997. A descriptive analysis and distribution frequency of the commonest causes of uterine rupture in 37 cases are presented taking into account medical, reproductive, health services provided and sociodemographic factors.

Results: Incidence of uterine rupture in Yemen was found to be (0.63), obstructed labor $83 \%$, contracted pelvis $19 \%$, previous surgery in $48 \%$, Oxytocine infusion in $42 \%$. Grand-multiparty was in $65 \%$ and maternal age over 35 years in $50 \%$. Antenatal care was only in $34 \%$.

Conclusion: The high percentage of malpresentation, cephalopelvic disproportion, previous uterine surgery accompanied by the high percentage of use of Oxytocin in this study highlights very clearly the role of this medication in increasing the risk of uterine rupture in Yemen.

\section{INTRODUCTION}

Uterine rupture is a life threatening obstetrical emergency encountered infrequently in the emergency department where the diagnosis is often missed or delayed, leading to maternal and fetal mortality and/or morbidity ${ }^{1}$. Previous analysis of obstetric factors as the cause of maternal death in Yemen shows that, the main causes were hemorrhage, ruptured uterus, eclampsia and puerperal sepsis ${ }^{2}$. Maternal mortality (100-800 per 100,000 live birth) and morbidity is still high in Yemen ${ }^{3}$. Because of the contribution of uterine rupture to maternal morbidity and mortality and because of the way its occurrence affects the life of women and family it is of a high priority in Yemen and other countries all over the world.

Of the estimated half million maternal deaths worldwide each year, only 6000 take place in developed countries. At the same time for every maternal death there are estimated to be more than fifteen women surviving pregnancy and childbirth with sever damage to their health such as vesico-vaginal fistula and pelvic inflammatory disease $^{4-7}$.
High maternal mortality and morbidity rate is a consequence of poor maternal care, inadequate socioeconomic and environmental conditions, poor accessibility to health services and poor nutrition habits. Contributing factors are also extremes of maternal age (too young or too old) and too many births within short intervals which cause fertility rate of 7.4 births per woman ${ }^{8}$.

About 20 percent of the population in developing countries is women of reproductive age. These women face one of the catastrophic risks of pregnancy "uterine rupture". Studies conducted in the developing world give strong evidence that uterine rupture is a major health problem in these countries with the rate being high in rural areas $^{9,10}$. They also reveal that socio-economic and cultural factors and shortcomings in health services play major roles in determining the frequency of uterine rupture. However, many efforts in developing countries have proven insufficient and ineffective in reducing maternal mortality in general because the issue is viewed as a medical or clinical dilemma rather than a broader public health problem ${ }^{11}$. Many programs thus focus only on biomedical determinants and ignore the complexity of the problem. 
The main reason for failure to consider socio-economic, culture and health determinants as causes of uterine rupture is the insufficient information on their contribution to the risk of uterine rupture.

According to the World Health Organization ${ }^{7}$ "exploring factors which are beyond the biomedical causes will have essential implication for preventive programs in the developing countries”.

Yemen has negligible amount of information on the determinants of uterine rupture. A study done by the Ministry of Health in Yemen during 1987 and 1989 showed that $14.2 \%$ of maternal deaths were caused by uterine rupture. The Central Planning Organization ${ }^{12}$ set a target to reduce maternal mortality by $50 \%$ by the Year 2010 wish dose not seam to be a reality.

Lack of information on maternal mortality and its causes, as Graham ${ }^{13}$ correctly points out, is "both a cause and effect of the more general neglect of maternal health". A clearer picture emerged from a number of specific studies that led to the international Safe Motherhood Initiative and call for action to improve maternal health ${ }^{14}$. One of the actions emphasized at the Nairobi Safe Motherhood conference was the need to carry out studies on levels and overall causes of maternal mortality in order to gain reliable country-specific information.

The causes of uterine rupture in Yemen can't be defined on medical bases alone. There are causes behind causes. A woman in Yemen will have uterine rupture because she is a grand-multipara in poor general health, which did not know about contraceptives, has no access to the family planning services and did not receive adequate and timely treatment. Generally it is because she is illiterate, poor and lives in a rural area.

The prenatal care in Yemen is very poor. According to the last published data only $44 \%$ of pregnant women had ever been to any prenatal clinic, with the frequency of visits during a pregnancy ranging from one to four. Women visit antenatal clinic mostly when they encounter a complication and very rarely for a routine antenatal care (13\%). $56 \%$ of pregnant women in Yemen have never had any antenatal care. Home delivery is still common in Yemen. About $78 \%$ of women deliver at home, $16 \%$ at state hospitals and about $5 \%$ at private hospitals. The home deliveries are usually attended by midwifes with minimal training, or relatives who have had some experience of labor. Some of the women will still deliver at home completely alone ${ }^{15}$.

The purpose of this study was to estimate the incidence and determine the risk factors of uterine rupture during pregnancy and delivery from both the medical and socio-economic points of view and to determine maternal and fetal outcomes among women using the referral hospital Al-thawra in Sana'a City, Yemen Rep. The aim was to define the causes and outcomes of this event and to suggest possible solutions and develop potential strategies for the reduction of uterine ruptures in the Republic of Yemen.

\section{METHODS AND MATERIALS}

The study was conducted in the Al-thawra hospital, the main hospital in Sana'a City, the capital of the Republic of Yemen. The hospital is a governmental one and charges only a nominal fee for services provided to the public. It is a referral hospital that receives cases from over the whole country. It has an average of about 8000 deliveries per year, 900 of them Caesarean sections performed for different reasons, one of which being uterine rupture. A retrospective study design was chosen. The data on women with uterine rupture delivering in Al-thawra hospital in the 9 months between September 1996 to May 1997 were collected by a hospital survey instrument. All cases $(n=37)$ of uterine rupture that arrived to or occurred in the hospital were collected, interviewed, examined and followed up. Complete information was obtained from all respondents, giving a $100 \%$ response rate. Women mostly presented to the obstetric emergency department. The history and examination of the patient was taken as situation allowed. All patients were followed up until their discharge from the hospital. Adhering to confidentiality safeguards the risks, benefits, objectives and procedures of the study were explained to study participants. Their informed consent was obtained verbally. The approval to conduct the study was obtained from the Department of Gynecology and Obstetrics of Sana'a University Yemen Republic and Palacky University, Faculty of Medicine, Gynecology and Obstetric Department Olomouc, Czech Republic. A combined Islamic and Gregorian calendar was prepared for accurate calculation of gestational age. The questionnaire covered the following information:

Medical problems, reproductive characteristics, health services provided, socio-demographic characteristics, the presenting complain, type of operation, fetal outcome and follow up characteristics. A comprehensive review of completed hospital questionnaires was performed and data base information was prepared. The variables were coded by their consequences on the questionnaire. Experts from the Statistical Department of Palacky university, medical faculty, performed data cleaning, entering and analysis using the Statistical Package for the Social Science (SPSS).

\section{RESULTS AND DISCUSSION}

The incidence of uterine rupture in this study was $0.63 \%$, (37 in 5877 deliveries) which is similar to or even other developing countries - $0.57 \%$ in Ethiopia ${ }^{16}$ and $0.45 \%$ in Morocco ${ }^{17}$. The incidence in developed countries is at least ten times lower $-0.086 \%$ in Australia ${ }^{18}$ and $0.023 \%$ in Ireland ${ }^{19}$. In the Czech Republic the incidence of uterine rupture is lower still when recorded according to the WHO recommendations. In the Czech Republic uterine rupture accompanied with serious postpartum hemorrhage necessitating hysterectomy occurs on average in 50 deliveries a year with a rate of 90000 deliveries per year (Štembera 2003, personal information). 
Risk factors of uterine rupture in this study can be divided into two main groups:

1. General, which can cause rupture of the uterus anywhere over the world.

2. Specific, that is more related to Yemen and maybe other developing countries.

General risk factors usually have a direct influence on pregnancy and delivery as for example medical and reproductive factors. Specific risk factors are those that we can call "causes behind the causes" through their indirect effects causing rupture of the uterus. They are mainly socio-demographic factors such as for example education, income and habits. An overlapping of these groups of factors could also occur as in the case of consanguinity and congenital anomalies. First-degree consanguinity, which was found in almost one third of study cases, is a sociodemographic indirect cause as well as a direct general risk factor. It increases the probability of congenital anomalies which in turn may cause uterine rupture associated with fetal death and serious maternal mortality and morbidity.

Obstructed labor due to cephalopelvic disproportion and malpresentation is one of the major direct factors for uterine rupture. Obstructed labor was found in $83.3 \%$ and contracted pelvis in $19.4 \%$ of cases. The malpresentation was very difficult to diagnose in some cases due to the uterus being already ruptured on admission. Obstructed labor can cause up to $93 \%$ uterine rupture as was reported in Ethiopia ${ }^{20}$.

Previous surgery of uterus could be a direct or indirect obstetrical cause for uterine rupture. Previous Caesarean section is still an important primary cause in Yemen. Seven cases $(19.5 \%)$ of uterine rupture in this study had a history of Caesarean section, one of which was "classical". This shows that this kind of operation is still performed in some cases ${ }^{21,22}$. Other previous surgeries were mainly curettage $(22.2 \%)$. It could be concluded that $47.5 \%$ of our patients had a surgical interference, which is considered a risk for rupture of the uterus.

Traumatic delivery was recorded in three cases $(8.3 \%)$ following vacuum extractor. It can be a potential risk for uterine rupture and should be considered as a possible cause $^{23,24}$.

Oxytocin infusion and prostaglandin vaginal application are other direct obstetrical risk factors for uterine rupture $^{25,26}$. This study showed a high percentage of its use; $41.7 \%$ of the respondents were given this drug (all but one had it through intravenous infusion). Oxytocin was used for augmentation of already prolonged and obstructed labor rather than for active management of labor concluding that the supervision and control over this drug is missing. Trial of labor in previously scarred uteri following CS can be safe when observing a number of rules but unfortunately this study showed that these rules were often missing ${ }^{22,21}$. The previous uterine surgery especially previous Caesarean section and the augmentation of labor by oxytocin are interacting risks. Oxytocin will rarely cause rupture on an unscarred uterus in the absence of other risks. If properly used even with scarred uterus can prevent repeated surgery and in this way reduce the risk in future pregnancies.

In general, the high percentage of malpresentation, cephalopelvic disproportion, previous uterine surgery accompanied by the high percentage of use of oxytocin in this study highlights very clearly the role of this medication in increasing the risk of uterine rupture. There is still a very little control over availability of medications in Yemen; a drug like Oxytocin can be sold to any person in some areas and can be used by any unskilled birth attendant.

Age and parity are interrelated risk factors. Parity is still a predisposing factor in Yemen, a country of a high fertility rate $(6,5)$ it could be one of the sole factors for uterine rupture ${ }^{27,28,29}$. This study has documented a very high parity among respondents. Only $35.1 \%$ had 1 to 4 children with the rest $(64.9 \%)$ having more than 5 up to 13 . Age of women in this study ranged from 18 to 42 years, with a mean \pm SD of 32.5 ( \pm 6.5 years $)$. Only one woman was 18 , almost one half of women were between 20 and 32 and more than half of the women were between 35 and 42 years old. Women older than 35 and women having their fifth or later birth are at greatest risk for uterine rupture and the importance of fertility regulation and contraception usage could be clearly concluded.

Antenatal care in this study shows that more than two thirds of the respondents $(76.0 \%)$ had never had any type of antenatal care during this pregnancy. The rest attended antenatal care to a different extent indicating poor health services (informational, financial or physical) that played a major role as a risk factor for uterine rupture. This study confirms the importance of this factor as it was also stated in the literature $11,30,31$.

One reason for coming late to the hospital could be the miscalculation of gestational age by the pregnant woman herself. At admission, $89 \%$ patients had a full term gestational age when calculated by the obstetrician compared with only $75 \%$ when calculated by the mothers.

The quality of curative health care and prevention in the study hospital was evaluated through some factors such as operating time, type of operation, duration of hospitalizations, complications after the operation, etc. It could be concluded that the quality was good enough to deal with the emergency cases.

Difficulty performing an exact diagnosis at the arrival of the patient with a severe diagnosis like uterine rupture may worsen the condition. In this study primary diagnosis has shown the lowest diagnostics reliability by abdominal examination (24.2\% was diagnosed as impending rupture, $24.2 \%$ as incomplete rupture and $48.5 \%$ as complete rupture). The reliability was increased by further vaginal examination (only $14.7 \%$ diagnosed as impending, $38.2 \%$ as incomplete and $47.1 \%$ as complete). The actual diagnosis made during the operation revealed an incomplete rupture in $42.9 \%$ of cases and a complete rupture in $57.1 \%$ giving a clear picture of the grave situations.

Blood availability and transfusion in a catastrophic event like uterine rupture is a life saving factor. This study had shown that a common scenario is that of an already 
anemic patient with heavy bleeding and poor availability of blood transfusion, which is a problem in nearly all the hospitals of Yemen. Bleeding in already anemic patient is still a further aggravating risk factor. Leaving the hospital being anemic followed by a pregnancy in short time can clearly explain the poor health of the mothers. Only $57.1 \%$ patients in the study had transfusions of 500-1000 ml of blood before and during the operation, though a majority of them (54.3\%) had already low hemoglobin level of $7-9 \mathrm{~g} / \mathrm{d}$ l before the operation. No wonder that just before the discharge the percentage of those with hemoglobin level of $7-9 \mathrm{~g} / \mathrm{dl}$ increased to $81.8 \%$. Levels of hemoglobin below $7 \mathrm{~g} / \mathrm{dl}$ were even recorded before the operation as well as just before the discharge. Blood availability is a real big problem in Yemen; blood donors are very rare. This hospital is trying to improve blood supply through obligating patient's relatives to give blood to replace the volume the patient is going to get.

The site of rupture of the uterus with its extension to the cervix (25.7\%) and rupture of the bladder (8.6\%) is in some cases a finding suggesting malpresentation, obstructed labor and late coming to hospital.

Fetal outcome was, death in $54.3 \%$ and survival in $45.7 \%$ with different degree of fetal compromise. In $45.7 \%$ of the cases fetuses were in the abdomen and $37.1 \%$ had the placenta in abdominal cavity also.

Fetal weight is a risk only when it contributes to cephalopelvic disproportion. That was not a predicting character in this study. Only in one case the fetal weight was more than $3500 \mathrm{~g}$.

Medication following the operation was used in a high percentage of patients. The type of medication used indicates the emergency and complications the respondents came with. Antibiotics were used in $100 \%, 32.4 \%$ received antigasgangren and $32.4 \%$ received antitetanic serum. Shock was present in the majority (70-80\%) of respondents arriving to the hospital. This shows that home deliveries are the preferred option and that patients usually came to the hospital only when they are suffering some complication and as an emergency. Most of the patients gave signs of obstructed labor and even of uterine rupture.

Maternal outcome in this study showed no mortality. Almost half of patients $(45.7 \%)$ had a repair of the rupture with tubal ligation, $14.3 \%$ had total hysterectomy, and $5.7 \%$ had subtotal hysterectomy. Therefore only $31.4 \%$ of patients could maybe reserve their fertility. Loss of fertility in Yemen is a catastrophic event; the infertile woman is rarely accepted and usually disgraced. Moreover, there is the possibility that her husband may divorce her or marry another wife.

Being a housewife in Yemen is an over-demanding work especially in rural areas with big families, grandmultiparity, malnutrition and heavy work, mainly carrying heavy things like water and wood for long distances since young age (two cases) affect the development of her stature and pelvis ${ }^{32}$. Many of these mothers, in addition, would have suffered malnutrition and vitamin $\mathrm{D}$ deficiency during childhood, predisposing them to osteomalacia, bone deformity and short stature. This could later lead to cephalopelvic disproportion and obstructed labor. Early marriages and starting childbearing before the maternal pelvis has attained its full potential size is another risk factor in an already malnourished mother.

Chewing Qat (an amphetamine like substance) to a high degree (44.4\%) among respondents as well as tobacco smoking $(25 \%)$ is having an indirect effect on women's nutritional status. It increases the chances of a malnourished mother to deliver a low birth-weight baby. According to UNICEF (1992) women who start life, as low birth-weight babies are more likely to suffer birthrelated mortality, especially from ruptured uterus due to pelvic disproportion, which is most often traced back to childhood malnutrition.

Socio-demographic determinants, such as rural/urban residence, women's education or family income and cultural influences are variables indirectly associated with uterine rupture through their effect on the health behavior of women and their health status. The majority of respondents $(83.8 \%)$ were completely illiterate. Over a half of respondents' husbands were farmers (40.5\%) and $27.0 \%$ were not working at all. Family income in $66.7 \%$ was between 10 and 25 thousands Yemeni Riyals (about 70 and 175 US \$) per month, with $30.6 \%$ below and $2.7 \%$ over this income range. The effect of these factors is well documented in literature.

\section{CONCLUSION AND RECOMMENDATIONS}

The causes of uterine rupture are complex, reflecting a combination of medical, reproductive, health services, nutritional and socio-economic factors. Studies of uterine rupture reveal that common direct medical causes are obstructed labor, previous Caesarean section scar specially if associated with the use of oxytocin or prostaglandin without proper care. Traumatic delivery and congenital uterine anomalies are also relatively common direct medical causes. Previous uterine surgery is the commonest indirect cause. The age and parity pattern on uterine rupture have shown the highest rate on one extreme (right) side of the typical "U" shape curve used to describe maternal mortality. Malnutrition, especially vitamin D deficiency, the lack of access to health services and socio-economic statuses are also essential factors for uterine rupture.

The recommendation can be classified into short term that can reduce the event of uterine rupture in Yemen by the time they will be applied and long term that will reduce the over all maternal mortality and morbidity all over Yemen including those due to the uterine rupture.

Early and adequate care for pregnant women, identifying predisposing risk factors must be taken into account before applying oxytocin. General improvement of the overall socio-economic conditions, better organization of the access to health services and better equipment of the medical centers and retraining of medical personnel is a prerequisite for any improvement. Educating the women and the whole population would greatly help in solving the problem. 
Finally, it is important to remember that in order to benefit mothers and children in the traditional society of Yemen, it is necessary that this and any future research is conducted within the framework of religious and traditional norms of the Yemeni society.

\section{REFERENCES}

1. Suner S, Jagminea L et al. Fatal spontaneous rupture of a gravid uterus: case report and literature. J Emerg Med, 1996; 14:181-5.

2. Abdulghani N. Risk factors for maternal mortality among women using Hospitals in North Yemen. A Thesis presented for the degree of PhD. in the Faculty of Medicine University of London, 1993.

3. Central Statistical Organization. The Second Population Policy Conference: Yemen Republic, National population commission, Sana'a, 1996.

4. Belsey M, Roysotn E. Overview of the Heath of Women and Children. Paper prepared for International Conference on Better Health for Women and Children through Family Planning, Nairobi, 1987; 5-9 October 1987

5. Mahler H. The Safe Motherhood Initiative: A call for action. The Lancet. 1987; 21:668-670.

6. Kindig M, Cardwell M. Delayed postpartum uterine dehiscence. A case report. J Reprod Med; Jul, 1998; 43:591-2.

7. World Health Organization. Studying Maternal Mortality in Developing Countries: Rates and Causes. WHO document FHE/ 87.7 Geneva: WHO, 1987.

8. UNICEF. The Situation of Children and Women in Rep. of Yemen, Sana'a, 1992.

9. UNICEF. The State of the World's Children Report Oxford University, Press New York, 1996.

10. Raczynski A et al. An assessment of the incidence of hemorrhage as a cause of maternal mortality in Poland 1985-1990. Acta Obstet et Gynecoloica, 1997; 76:24.

11. Campbell, Graham. Measuring the determinants of materna morbidity and mortality: Defining and selecting outcomes and determinants, and demonstrating association. Maternal and child epidemiology unit publication, London: London School of Hygiene and Tropical Medicine, 1991b.

12. Central Planning Organization. The National Population and Housing. Sana'a Yemen Arab Republic, 1990a.
13. Graham WJ, Campbell OMR. Measuring maternal health: defining the issues. Maternal and Child Epidemiology Unit Publication. London: London School of Hygiene and Tropical Medicine, 1991.

14. The Lancet. The Safe Motherhood Initiative: A Call to Action. March; 1987; 21:668-670.

15. Yemen family health survey, principal report. League of Arab states social sector, 2005

16. Chamiso R. Uterine rupture in Shashemene General Hospital, South Shoa. Ethiop Mwd J: 1995; 33:251-7.

17. El Mansouri A. Accouchements sur uterus cicatriciel. Apropos de 150 cas. Rev Fr Gyencol Obstet, 1994; 89:606-12.

18. Lynch JC, Pardy JP. Uterine rupture and scar dehiscence. A fiveyear survey. Anaesth Intensive care. 1996; 24:704.

19. Gardiel F, Daly S, Turner Mj. Uterine rupture in pregnancy reviewed. Eur J Obstet Gynecol Report Biol. 1994; 56:10.

20. Deneke F. Brief communication, Ruptured uterus in Ethiopia. Int J Gyn Obst, 1996; 54:175-176.

21. Abbassi $\mathrm{H}$ et al. Accouchement des uterus cicatriciels: pet-on elargir l'epreuve uterine. Gynecol Obstet Biol Reprod, 1998; 27:452-9.

22. Cowan KK et al. Trial of labor following Caesarean delivery. Obstet Gynecol, 1994; 83:933-6.

23. Ferguson RK, Reid DE. Rupture of the uterus: A twenty-year report from the Boston Lying-in Hospital. Am J Obstet Gynecol, 1958; 76:172-80.

24. Miller et al. Rupture of the unscarred uterus. Obstetric and Gynecology, 1997; 89:671-673.

25. Azem $F$ et al. Uterine rupture with the use of a low-dose vaginal PGE2 tablet. Acta Obstet Gynecol Scand, 1993; 72:316-7.

26. Phuapralit $\mathrm{W}$ et al. Uterine rupture and labor induction with prostaglandins. J Med Assoc Thai, 1993; 76:292-5.

27. Sweeten KM, Graves WK, Athanassiou A. spontaneous rupture of the unscarred uterus. Am J Obstet Gynecol, 1995; 172:1851-6.

28. Khan NH. Rupture of the uterus. JPMA J Pak Med Assoc, 1993; 43:174-6.

29. Longombe AO, Lusi KM, Nickson P. Obstetric uterine ruptures in rural area in Zaire. Trop Doct, 1994; 24:90-3.

30. Mulumba N. Rupture of the uterus. BMJ, 1996; 312:1204-5.

31. Zanconato $\mathrm{G}$ et al. Audit of uterine rupture in Maputo: a tool for assessment of obstetric care. Gynecol Obstet Invest, 1994; 38:151-6.

32. World Health Organization. Monitoring of Reproductive Health: Selecting short list national and global indicators. 1997. 